\title{
VAN DER WAALS' ATTRACTION BETWEEN ANISOMETRIC PARTICLES
}

\author{
Marjorie J. Vold ${ }^{1}$ \\ Van't Hoff-Laboratory, University of Utrecht, Netherlands \\ Received May 27,1954 \\ SUMMARY
}

Calculations based on additivity of the London-van der Waals' dispersion forces show that for particles of colloidal dimensions the total attractive energy is of the same order of magnitude as thermal energies when the mean diameter $\left(v^{1 / 3}\right.$, where $v$ is the volume) is of the order of magnitude of the particle separation, regardless of whether the particles are ball-, rod-, or plate-shaped. At smaller separations the order of attractive energies is plates $>$ rectangular rods $>$ cylinders $>$ spheres. At separations such that $V \sim 10 k T$ the attraction between spheres varies nearly as $r^{-1}$, but for rods and cylinders it varies approximately $r^{-2}$ and for platelets $r^{-3}$.

likewise, (for rods) parallel orientation is greatly favored over perpendicular orientation at small separations, and (for rectangular rods) orientation with the largest faces opposite each other is favored. These differences diminish as the particle separation increases but remain important so long as the van der Waals' attraction itself is of the order of $k T$ or larger.

Although the essential features of the London-van der Waals' forces of interaction of colloidal particles are widely recognized, actual calculations appear to have been made only for spherical particles and parallel plates whose area is large compared to their separation (3). The result of Dube and Das Gupta (1) for disclike particles does not appear to be useful, because the limitation of infinite thinness for the dises in the beginning of the calculation means that the limiting formula for "small" distances can be valid only for distances large compared to the disc thickness. In fact, for parallel discs perpendicular to the line of centers they find an inverse fourth-power law, which is similar to that found for platelike particles when their separation is large compared to their thickness (5). One factor contributing to the lack of ealculations for various shapes of particles is the intractability of the pertinent integral

$$
V=-\int_{v_{1}, v_{2}} \frac{q^{2} \lambda d v_{1} d v_{2}}{r^{6}}
$$

1 Guggenheim Fellow at the University of Utrecht.

Permanent address (after September 1, 1954): Department of Chemistry, University of Southern California, Los Angeles, Calif. 

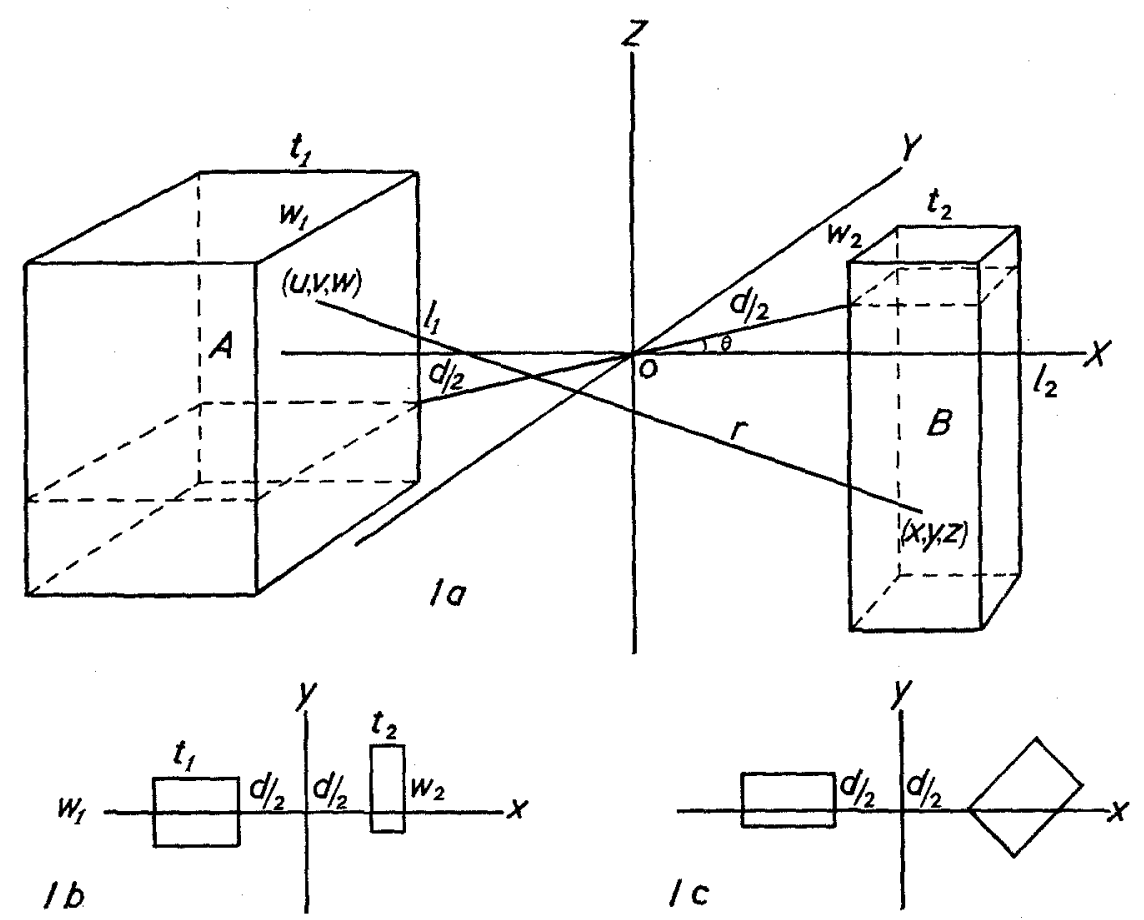

FIg. 1. Nomenclature and co-ordinate system. The two particles $A$ and $B$ are parallel but otherwise unrestricted in relative positions. The origin is always located so as to bisect the shortest distance between them which may be between corners as in Fig. Ia or sides as in Fig. 1b. In the calculations at least one dimension of each particle has been assumed large compared to the others and to the separation. Moreover, the explicit formulas are given for identical particles whose ends (cross section $t, w)$ lie in the same plane. Figure $1 c$ shows the relative orientation which has not been successfully treated.

where $V$ is the total attractive energy, $v_{1}$ and $v_{2}$ are the volumes of particles containing $q$ atoms per unit volume whose London constant is $\lambda$, and $r$ is the distance between a point in one particle and a point in the other.

However, explicit integration of [1] can be carried out for rectangular rods so oriented that the edges are parallel to the $x, y$, or $z$ axes of a cartesian co-ordinate system, and the resulting attractive energy expressed as a function of the Hamaker constant, $\pi^{2} q^{2} \lambda$, the dimensions of the particles, and $d$, the smallest distance between them, provided the rods are long compared to their cross section and their separation. Such a shape is more nearly representative of the actual shape of many real particles (soap fibers in both aqueous gels and greases, linear polymers in extended configurations, rod-shaped protein molecules, etc.) than either of the previously treated cases.

Figure 1 shows two such rectangular particles, the co-ordinate system, and the nomenclature in terms of which Eq. [1] can be written 


$$
V=-q^{2} \lambda \iiint \iiint \frac{d x d y d z d u d v d w}{\left[(x-u)^{2}+(y-v)^{2}+(z-w)^{2}\right]^{3}} .
$$

Two of the six integrations can be performed at once, leading to the result:

$$
\begin{aligned}
V=-\frac{q^{2} \lambda}{8} \iiint \int d x d y d u d v \\
\cdot\left[\frac{1}{R^{2}\left[R^{2}+(z-w)^{2}\right]}-\frac{3(z-w)}{R^{5}} \tan ^{-1} \frac{z-w}{R}-\frac{3 \ln R}{R^{4}}\right]
\end{aligned}
$$

where $R^{2}=(x-u)^{2}+(y-v)^{2}$. Further progress requires insertion of the limiting values of $(z-w)$. From Fig. $1 a$ for identical particles whose ends lie in the same plane, $(z-w)=0$ or $\pm l$ so that.

$$
V=-\frac{q^{2} \lambda}{4} \iiint \int d x d y d u d v\left[\frac{1}{R^{4}}-\frac{1}{R^{2}\left(R^{2}+l^{2}\right)}+\frac{3 l}{R^{5}} \tan ^{-1} \frac{l}{R}\right] .
$$

Further progress appears to present difficulties unless an expansion be made in powers of $R / l<1$, which leads to

$$
V=-\frac{q^{2} \lambda}{4} \iiint \int d x d u d y d v\left[\frac{3 \pi l}{2 R^{5}}-\frac{2}{R^{4}}+\sum_{n=0}^{\infty}(-1)^{n} \frac{2(n+1) R^{2 n}}{(2 n+5) l^{2 n+4}}\right] .
$$

The second term here is smaller than the first by a factor of $4 R / 3 \pi l$, and the third is smaller than the second by $1 / 5(R / l)^{4}$. Now $R_{\max }$ is of the order of $\left(w^{2}+t^{2}+d^{2}\right)^{1 / 2}$, where $w$ is the width, $t$ the thickness, and $d$ the separation of the particles. It follows that calculations based on only the first term are valid for particles of mean diameter small compared to their length and separated by distances which are also small compared to their length. Although the calculations of the present paper are based only on. this term, the second and higher terms could be included without real difficulty whenever either experimental data or further theoretical developments justify the labor involved.

Keeping only the first term, the next two quadratures lead to

$$
V=-\frac{\pi q^{2} \lambda l}{8} \iint \frac{d y d v\left[2(x-u)^{2}+(y-v)^{2}\right]}{(y-v)^{4}\left[(y-v)^{2}+(x-u)^{2}\right]^{\frac{1}{2}}}
$$

in which limiting values of $(x-u)$ are still to be inserted. These will depend upon the mutual orientation of the particles about their long axes. When $(x-u)=0$ the form taken by Eq. [3] is

$$
V=-\frac{\pi q^{2} \lambda l}{8} \iint \frac{d y d v}{(y-v)^{3}}=\frac{\pi q^{2} \lambda l}{16(y-v)}
$$

while for $(x-u)=$ constant $=k$

$$
V=+\frac{\pi q^{2} \lambda l}{24} \frac{\left[k^{2}+(y-v)^{2}\right]^{3 / 2}}{k^{2}(y-v)^{2}}
$$


with $(x-u)=k$ very large

$$
V=+\frac{\pi q^{2} \lambda l k}{24(y-v)^{2}} .
$$

Formulas for the total potential for various orientations can now be written down by inserting the appropriate limits in Eqs. [4]. For the particle orientation illustrated in Fig. $1 b$ the formula is

$$
\begin{aligned}
& \dot{V}=- \frac{\pi q^{2} \lambda l}{12} \\
& \cdot\left\{\frac{\left(w^{2}+d^{2}\right)^{3 / 2}}{w^{2} d^{2}}+\frac{\left(w^{2}+(d+2 t)^{2}\right)^{3 / 2}}{w^{2}(d+2 t)^{2}}-\frac{2\left[w^{2}+(d+t)^{2}\right]^{3 / 2}}{w^{2}(d+t)^{2}}\right\} \\
& \quad+\frac{\pi q^{2} \lambda l}{8}\left[\frac{1}{d}+\frac{1}{d+2 t}-\frac{2}{d+t}\right] .
\end{aligned}
$$

For small values of the separation this formula yields

$$
V=-\frac{\pi q^{2} \lambda l w}{12 d^{2}}
$$

and for large values

$$
V=-\frac{3 \pi q^{2} \lambda l w^{2} t^{2}}{8 d^{5}}
$$

For case [1a], the formula is

$$
\begin{aligned}
& V=-\frac{\pi q^{2} \lambda l}{24}\left\{\frac{1}{d \cos ^{2} \theta \sin ^{2} \theta}+\frac{4\left[(q+t)^{2}+(p+w)^{2}\right]^{3 / 2}}{(q+t)^{2}(p+w)^{2}}+\frac{\left[(q+2 t)^{2}+p^{2}\right]^{3 / 2}}{(q+2 t)^{2} p^{2}}\right. \\
& +\frac{\left[(p+2 w)^{2}+q^{2}\right]^{3 / 2}}{(p+2 w)^{2} q^{2}}+\frac{\left[(p+2 w)^{2}+(q+2 t)^{2}\right]^{3 / 2}}{(p+2 w)^{2}(q+2 t)^{2}}-\frac{2\left[(q+t)^{2}+(p+2 w)^{2}\right]^{3 / 2}}{(q+t)^{2}(p+2 w)^{2}} \\
& -\frac{2\left[(p+w)^{2}+(q+2 t)^{2}\right]^{3 / 2}}{(p+w)^{2}(q+2 t)^{2}}-\frac{2\left[(p+w)^{2}+q^{2}\right]^{3 / 2}}{(p+w)^{2} q^{2}}-\frac{2\left[(q+t)^{2}+p^{2}\right]}{(q+t)^{2} p^{2}}
\end{aligned}
$$

where for convenience $p=d \sin \theta$ and $q=d \cos \theta$. For large distances this has the same limit, as Eq. [5] but for small distances the limit is

$$
V=-\frac{\pi q^{2} \lambda l}{24 d \cos ^{2} \theta \sin ^{2} \theta} .
$$

Finally, the interaction energy for two rods crossed perpendicularly with flat sides parallel to each other can be obtained by choosing $z= \pm \infty$ in [2] with $w= \pm w / 2$ and $u= \pm \infty$ in [3] with $x= \pm w / 2$. This gives

$$
V=-\frac{\pi q^{2} \lambda w^{2}}{12}\left[\frac{1}{d^{2}}+\frac{1}{(d+2 t)^{2}}-\frac{2}{(d+t)^{2}}\right] .
$$


Formulas [5] through [7] permit discussion of the mutual attraction as a function of particle separation and particle orientation, but the restriction on the length of the particles must not be forgotten.

\section{Particle Orientation}

Three cases have been considered: (1) the relative attractive energies between two ribbon-like particles as a function of whether their wide surfaces face each other $(w>t)$ or narrow surfaces face each other $(w<t)$; (2) the relative attractive energies between two symmetrical rods $(w=t)$ as a function of whether their flat sides face each other or their diagonals face each other $\left(\theta=45^{\circ}\right.$ in Eq. [6]); and (3) the relative attractive energies for perpendicular or parallel orientation of the rods.

It can be seen from formula [ $5 b]$ that for ribbon-like particles of constant cross section $(w, t)$ the attraction at large distances is independent of the orientation of the particles about their long axes. For small distances formula [5a] shows that the attraction is greater for wide sides facing each other, directly in the ratio of $w$ to $t$. Intermediate cases can be visualized more easily by numerical examples than by algebraic manipulation of $\mathrm{Eq}$. [5]. Figure 2, curve $A$ shows the ratio of $V_{w}$ (attractive energy for wide sides facing each other) to $V_{t}$ (narrow sides) for a ratio $w / t=10$ as a function of a distance parameter $\sigma=\left(d^{2} / w t\right)^{1 / 2}$. It can be seen that there is a strong preference for orientation with wide sides facing each other which persists out to quite large distances.

Comparison of formula [5a] with formula $[6 a]$ for $\theta=45^{\circ}$ shows that at small distances the attractive energy for parallel rather than diagonal orientation is larger by a factor of $w / 2 d(w / 2 d \gg \mathbf{1}$ for the validity of these formulas). Figure 2, curve $B$, which was calculated using Eqs. [5] and [6] (with $w=t, \theta=45^{\circ}$ ) shows $V_{p}$ (parallel orientation) $/ V_{d}$ (diagonal orientation) as a function of the distance parameter $d / w$. It can be seen that $V_{p}$ is very much larger, even for substantial distances. A somewhat more interesting case, in which the diagonal of one particle cross section is parallel to the edge of the second, can not be easily derived from Eq. [1a].

Comparison of formula [5a] with [7] shows that parallelism of the rods along their length is favored over perpendicular orientation by a factor of $l / w$ at small distances. Estimates of the rate at which this preference diminishes are not, as in the previous cases, independent of just how long the "infinitely long" particles assumed in the derivation are actually taken to be. Figure 2, curve $C$, which was calculated for a length/width ratio of 20 , shows that this preference persists up to a particle separation appreciably larger than the rod diameter.

\section{Particle Separation}

The total van der Waals' attraction between spheres depends only on the ratio of the separation to the diameter of the sphere. For large plate- 
lets or long rods, this is no longer true. The formula for thin platelets can be put in the form

$$
V_{P}=-\frac{A}{12 \pi s^{2}} \cdot \frac{v}{t^{3}}\left(1+\frac{s^{2}}{(s+2)^{2}}-\frac{2 s^{2}}{(s+1)^{2}}\right)
$$

where the parameter $s=d / t, A=\pi^{2} q^{2} \lambda$, and $v$ is the volume of the plates. The ratio $v / t^{3}$ can be looked on as an asymmetry factor. Hamaker's for-

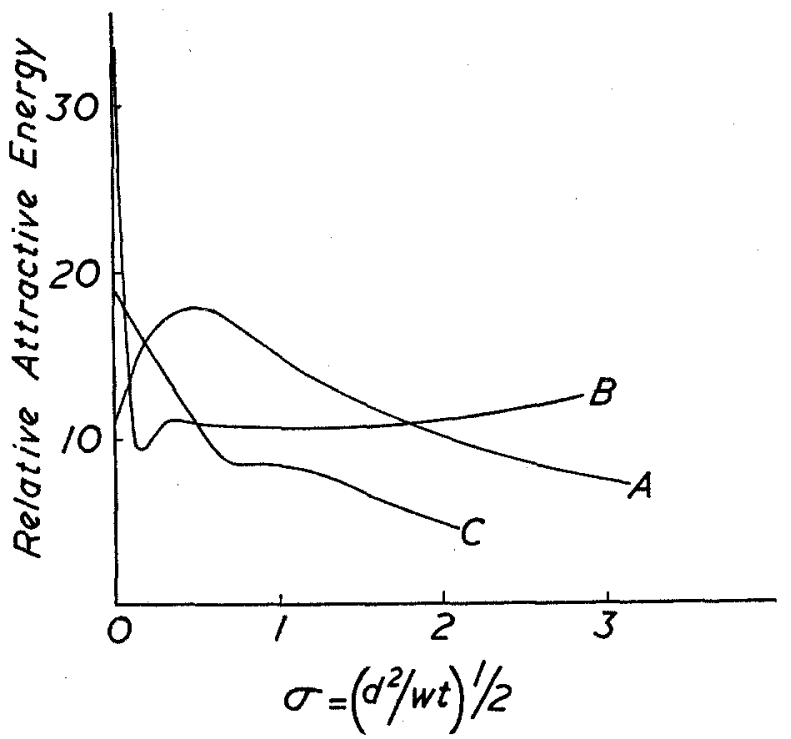

FIG. 2. Relative attractive energy between rod-shaped particles as a function of separation for various orientations. $A . V_{w} / V_{t}$ for laths for which $w / t=10 . B . V_{p} / V_{d}$ for rods of square cross section with $V_{p}$ corresponding to Fig. $1 b$ and $V_{d}$ to Fig. $1 a$. C. $V_{p} / V_{,}$for rods of square cross section with $V_{\rho}$ corresponding to long rods parallel and $V$, to long rods crossed perpendicularly, calculated for a length/width ratio of 20. All curves must approach unity for large enough $\sigma$, but $V$ itself is no longer important when $\sigma$ becomes as large as 2 or 3 where the orientation still produces differences of factors of 5 to 10 .

mula for equal spheres and Eq. [5] of this paper for rods of square cross section, in a similar form are

$$
V_{s}=-\frac{A v}{4 \pi s D^{3}}\left(\frac{2}{s+2}+\frac{2 s}{(s+1)^{2}}+4 s \ln \frac{s(s+2)}{(s+1)^{2}}\right)
$$

and

$$
\begin{array}{r}
V_{R}=-\frac{A}{12 \pi s^{2}} \cdot \frac{v}{t^{3}}\left\{\left(1+s^{2}\right)^{3 / 2}+\frac{s^{2}\left[1+(s+2)^{2}\right]^{3 / 2}}{(s+2)^{2}}-\frac{2 s^{2}\left[1+(s+1)^{2}\right]^{3 / 2}}{(s+1)^{2}}\right. \\
\left.-\frac{3}{2} s-\frac{3 s^{2}}{2(s+2)}+\frac{3 s^{2}}{s+1}\right\} \cdot \quad[10]
\end{array}
$$




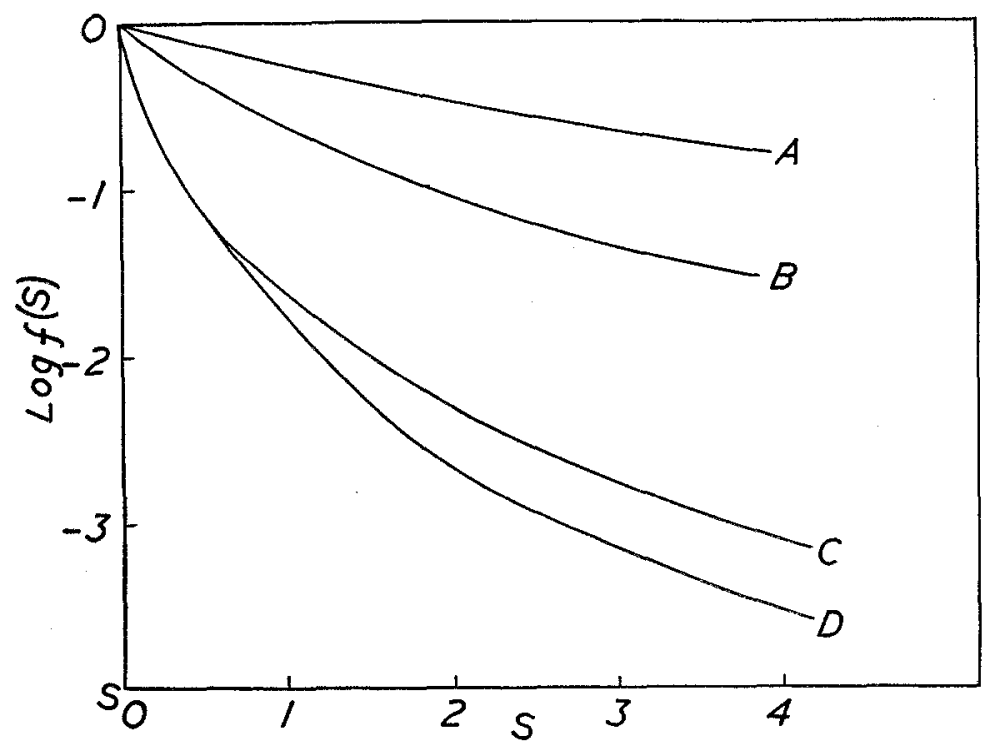

Fig. 3. $f(s)$ for various particle shapes. In every case the van der Waals' attraction can be expressed as a limiting law multiplied by a factor approaching unity at small distances, and decreasing rapidly at large distances, see Eqs. [8]-[11]. Curve $A \mathrm{~s}$ platelets. Curve $B$, rods of square cross section oriented as in Fig. $1 b$. Curve $C$, rodof square cross section oriented as in Fig. 1a. This orientation is regarded as approxi, mating the effect expected for cylindrical particles. Curve $D$, spheres.

Even though the discussion of orientation shows that the rods are more likely to be oriented in such a way as to make Eq. [5] rather than Eq. [6] applicable, Eq. [6] has been cast in the same form and included in the calculations on the grounds that it may represent a fair approximation to the behavior of cylindrical particles. For this case

$$
\begin{aligned}
V_{C}=-\frac{A}{3 \pi^{2} s} \cdot & \cdot \frac{2 v}{t^{3}}\left\{1+\frac{s}{s+2}+\frac{4 s}{s+1}+\frac{\sqrt{2}}{2} \frac{\left(s^{2}+(s+2)^{2}\right)^{3 / 2}}{s(s+2)^{2}}\right. \\
& \left.-\frac{\sqrt{2}\left(s^{2}+(s+1)^{2}\right)^{3 / 2}}{s(s+1)^{2}}-\frac{\sqrt{2} s\left[(s+1)^{2}+(s+2)^{2}\right\}^{3 / 2}}{(s+1)^{2}(s+2)^{2}}\right\}
\end{aligned}
$$

where $t$ is now the diameter of the "cylinder" and $s=d / t$. The four functions of the closely related parameters $(s)$ in formulas [8]-[11] have been plotted in Fig. 3, which is quite useful for further calculations.

The relative attractive energies of particles of equal volume at the same distance of closest approach is dependent upon just how large are the "infinite" platelets of formula [8] or how long are the "infinite" rods of formulas [10] and [11]. However, at small separations, when the functions of $s$ are nearly unity, the attraction between the anisometric particles is always larger than for spherical particles of equal volume, because the 


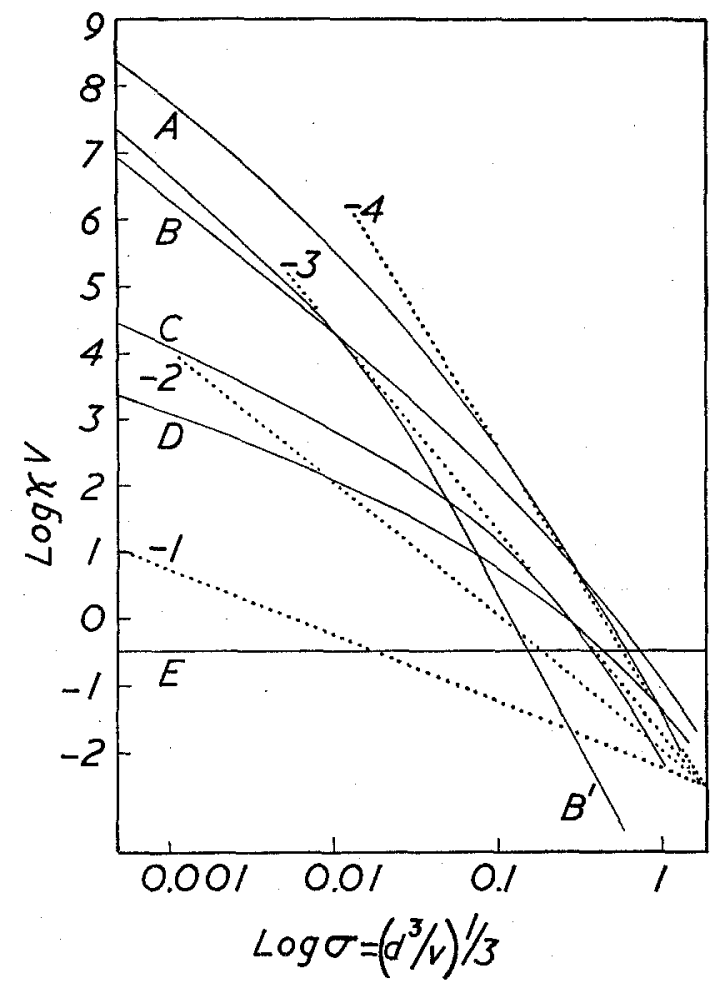

FIG. 4. Van der Waals' attractive energy between colloidal particles of the same volume but different shapes. Curve $A$, plates with an edge/thickness ratio of 800 . $B$ and $B^{\prime}$, rods of square cross section with length/width ratios of 28 and 316, respectively. Curve $C$, "cylinders" of length/diameter ratio of 28 , i.e., rods of square cross section oriented as in Fig. 1a with length/diagonal ratio of 28 . Curve $D$, spheres. Line $E, V=k T$ for $A=3 \times 10^{-12}$ and $k T=4 \times 10^{-14}$. Units: The ordinate is $\log$ $24 V / A$ where $V$ is the mutual van der Waals' energy in ergs and $A$ is Hamaker's constant. The abscissa (on a logarithmic scale) is the dimensionless ratio of the separation $d$ to the cube root of the volume $v$. The dotted lines of varying slope show how $V$ is varying with separation. For example, curve $A$ is parallel to the line of slope -2 at very small separations, slope -3 at $\sigma=0.05$, and slope -4 at $V=k T$.

thickness $t$ is always less than the diameter of the equivalent sphere and occurs in an inverse power. This result is seen to be plausible since, at a given distance of closest approach, a far higher proportion of the total number of atoms in the particles of a flat plate or rod are at or near this separation than is the case with spherical particles.

The order of magnitude of the differences is indicated by Fig. 4, which shows the course of the attractive energy as a function of a separation variable $\left(d / v^{1 / 3}\right)$ keeping $v$ constant. The platelets were taken to have an edge/thickness ratio of 800 , which is a not unreasonable approximation for bentonite particles, while the rods were taken to have a length/thick- 
ness ratio of 28 , which is reasonable for soap fibers, as estimated from electron micrographs. An additional curve is plotted for much more asymmetric rods $(l / t=316)$.

All the curves tend to convergence as the separation increases, as indeed they must, for at very large distances all must eventually approach the inverse sixth-power law valid for atoms. The important observation is that this convergence brings the attractive energies to the same rough order of magnilude, independent of shape and orienlation, when the separation approaches $v^{1 / 3}$. This observation is, of course, dependent upon arbitrary choices of particle asymmetry, and curve $A$ of Fig. 4 calculated for extremely asymmetric rods illustrates the point that for such cases the attractive energy is of shorter range. However, the choices made are reasonable approximations to the shapes encountered in real systems.

Recently (4) considerable attention has been given to the possible importance for coagulation phenomena of a so-called "secondary" minimum in the total potential energy curve for two particles at fairly large separations. It is not possible to make great progress in considering such minima for anisometric particles without a knowledge of the accompanying repulsive interactions. However, for such a minimum to have appreciable depth, $V_{\text {attr. }}$ must be of the order of at least $10 k T$ and perhaps larger. For attractive energies of this order of magnitude the form of the dependence of attractive energy on separation is quite different for different particle shapes, being steepest for platelets and most gradual for spheres, with rods and cylinders occupying an intermediate position. It follows that the depth and location of the secondary minimum may be quite dependent upon particle shape.

A second commonly observed phenomenon (2) is that when symmetrical particles aggregate to form flocs, the flocs are often asymmetric, like beads on a string. But the van der Waals' interaction between asymmetric particles is such that they tend on approach to orient themselves to build a more symmetrical double particle, so that it seems unlikely that the explanation of chain building by aggregation of isometric particles is to be found in the attractive term.

The writer wishes to thank Prof. J. Th. G. Overbeek for many helpful suggestions during the course of this development and the John Simon Guggenheim Foundation for financial support.

\section{REFERENCES}

1. Dube, G. P., And Das Gupta, H. K., Indian J. Phys. 13, 411 (1939).

2. Foster, M. J., And Mead, D. J., J. Appl. Phys. 22, 705 (1951).

3. Hamaker, H. C., Physica 4, 1058 (1937).

4. Overbeek, J. Tr. G., in Kruyt “Colloid Science," Vol. 1, p. 324. Elsevier, Amsterdam, 1952.

5. Verwey, E. J. W., and Overbeer, J. Th. G., "Theory of the Stability of Lyophobic Colloids," p. 101. Elsevier, Amsterdam, 1948. 